Southern Illinois University Carbondale

OpenSIUC

Articles

Department of Plant, Soil, and Agricultural Systems

2010

\title{
Iso-lines and inbred-lines confirmed loci that underlie resistance from cultivar 'Hartwig' to three soybean cyst nematode populations
}

Samreen Kazi

Jeffry Shultz

Jawad Afzal

Rizwan Hashmi

Mohammed Jasim

See next page for additional authors

Follow this and additional works at: http://opensiuc.lib.siu.edu/psas_articles

Published in TAG Theoretical and Applied Genetics, (2010) 120 (3): 633-644, http://dx.doi.org/ 10.1007/s00122-009-1181-4 The original publication is available at www.springerlink.com.

\section{Recommended Citation}

Kazi, Samreen, Shultz, Jeffry, Afzal, Jawad, Hashmi, Rizwan, Jasim, Mohammed, Bond, Jason, Arelli, Prakash R. and Lightfoot, David A. "Iso-lines and inbred-lines confirmed loci that underlie resistance from cultivar 'Hartwig' to three soybean cyst nematode populations." (Jan 2010).

This Article is brought to you for free and open access by the Department of Plant, Soil, and Agricultural Systems at OpenSIUC. It has been accepted for inclusion in Articles by an authorized administrator of OpenSIUC. For more information, please contact opensiuc@lib.siu.edu. 
Authors

Samreen Kazi, Jeffry Shultz, Jawad Afzal, Rizwan Hashmi, Mohammed Jasim, Jason Bond, Prakash R. Arelli, and David A. Lightfoot 
Isolines confirm both separate and common loci underlie the resistance from Glycine max cultivar 'Hartwig' to three soybean cyst nematode populations.

Theor Appl Genet (revision 8).

S. Kazi ${ }^{1,2}$, J. Shultz ${ }^{1,2,3}$, J. Afzal ${ }^{1,2}$, R. Hashmi ${ }^{1,6}$, M. Jasim ${ }^{1,7}$, J. Bond ${ }^{1}$, P.R. Arelli ${ }^{1,4}$ and D.A.Lightfoot ${ }^{1,2,5}$

Keywords:

nematode; resistance; soybean; Glycine max; Hartwig; SDS; SCN; cqSCN-005

1* Plant Biotechnology and Genomics Core-Facility, Department of Plant, Soil, and Agricultural Systems, Southern Illinois University, Carbondale, IL 62901, USA.

2 Center for Excellence in Soybean Research, Teaching and Outreach, Southern Illinois University, Carbondale, IL 62901, USA.

3 Present Address: School of Biological Sciences, Louisiana Tech University, Ruston LA, USA.

4 Present Address: Soybean Genetics Laboratory, USDA, ARS-MSA, Jackson TN 38301, USA.

${ }^{5}$ Corresponding author.

${ }^{6}$ Department of Plant Biology, Southern Illinois University, Carbondale, IL 62901,USA.

${ }^{7}$ Visiting Scholar: from Dept of Biotechnology, Al-Anbar Uni., Tehran, Iraq. 


\begin{abstract}
Soybean [Glycine max (L.) Merr.] cultivars vary in their resistance to different populations of the soybean cyst nematode $(\mathrm{SCN})$, Heterodera glycines. Populations are classified into groups called HG Types. The rhgl locus on linkage group G underlying resistance to HG Type 0 was necessary for resistance to all other HG types. However the loci for resistance to $H$. glycines HG Type 1.3- (race 14) and HG Type 1.2.5- (race 2) of the soybean cyst nematode may vary in their locations. The aims were to compare the inheritance of resistance to three nematode HG Types in a population segregating for resistance to $\mathrm{SCN}$ and to identify the underlying quantitative trait loci (QTL). 'Hartwig', a soybean cultivar resistant to most SCN HG Types was crossed with the susceptible cultivar 'Flyer'. A total of 92 F5-derived recombinant inbred lines (RILs) and 144 molecular markers were used for map development. The $r h g 1$ associated QTL found in earlier studies were confirmed and shown to underlie resistance to all three HG Types in RILs (Satt309; HG Type 0, P=0.0001 R $\mathrm{R}^{2}=22 \%$; Satt275; HG Type 1.3.., $P=0.001, \mathrm{R}^{2}$ $=14 \%$ ) and NILs (Satt309; HG Type 1.2.5-, P=0.001 $\left.\mathrm{R}^{2}=24 \%\right)$. A new QTL underlying resistance to HG Type 1.2.5- was detected on LG D2 (Satt574; $\mathrm{P}=0.001, \mathrm{R}^{2}=$ $11 \%$ ) among 14 RILs resistant to the other HG types and was confirmed in a small NIL population consisting of 60 plants of ten genotypes $(\mathrm{P}=0.04)$. This QTL (cqSCN-005) is located in an interval previously associated with resistance to both SDS leaf scorch (cqSDS-001) and SCN HG Type 1.3-. The QTL detected will allow marker assisted selection for multigeneic resistance to complex nematode populations in combination with sudden death syndrome resistance (SDS) and other agronomic traits.
\end{abstract}




\section{INTRODUCTION}

Heterodera glycines Ichinohe infestation of soybean fields leads to "Yellow dwarf" disease (Hussey and Grundler 1998; Davis et al. 2004). H. glycines is a small plant-parasitic microscopic roundworm that attacks the roots of soybean. The juvenile nematode that hatches from the egg is the infective stage of soybean cyst nematode ( $\mathrm{SCN})$, that enters the soybean root. $\mathrm{SCN}$ infections cause various symptoms including chlorotic (yellow) patches, root necrosis, suppression of root and shoot growth and reduced yield. Worldwide it is the most serious pest of soybean (Wrather et al. 1996; Wrather et al. 2003). Once established in a field, the infestation is difficult to eradicate due in the ability of the nematode populations to overcome soybean resistance genes.

The most economic and environmentally sound solution for managing areas infested with SCN is the development of resistant germplasm (Concibido et al. 2004). However the resistance is race-cultivar specific (H. glycines biotype, HG type; Niblack et al. 2003), temperature dependent (Palmateer et al. 2002) and pathogen populations have the ability to mutate, recombine and/or drift quickly to overcome resistance (Niblack et al. 2003).

In most germplasm, $\mathrm{SCN}$ resistance was shown to be controlled by few major QTL (Concibido et al. 2004). For example, in the cultivar 'Forrest', resistance to SCN HG Type 0 is a qualitative trait and has a specific 2 locus requirement for $r h g 1$ and $R h g 4$. (Meksem et al. 2001). The rhgl locus is on linkage group G and provides the major portion of resistance to SCN across many genotypes (Webb et al. 1995; 1996; Concibido et al. 1997; 2004). Varieties selected for rhgl provide good resistance to almost all HG types. Satt309 has been variously mapped at 0.5-2 cM distance from $r h g 1$, and is $42 \mathrm{Kbp}$ 
from a candidate gene cluster in the physical map (Ruben et al. 2006; Afzal and Lightfoot 2007; Afzal et al., 2008; 2009). Markers from within the gene cluster, like the indel marker TMD1 in the intron of a receptor like kinase (RLK), are very strongly associated with SCN traits. However, recombination events suggest the rhgl locus lies between TMD1 and Satt309, a $42 \mathrm{~kb}$ region encompassing genes encoding the kinase of the RLK, a laccase like protein and a transporter like protein (Ruben et al. 2006; Afzal et al 2008; 2009; Iqbal et al 2009)

The Rhg4 locus is on linkage group A2 (Weisemann et al. 1992; Webb et al. 1995; 1996; Meksem et al. 2001) and provides an equal portion of resistance to SCN HG Type 0, and in some crosses HG Type 1.3- (Webb et al 1995; 1996; Concibido et al. 1997; 2004). It does not contribute to resistance to HG Type 1.2.5-; or HG Type 2. Markers closest to the locus include the RFLP derived SCAR marker BLT65 (Weiseman et al., 1992), AFLP derived SCAR marker A2D8 (Meksem et al. 2001) and the BESSSRs from the BAC B100B10 anchored by A2D8 (Shultz et al. 2007). The BES-SSR H100B10b has been located in the soybean genome (Lightfoot, unpublished) in $35 \mathrm{kbp}$ region associated with the deletion underlying the $I$ gene for seed coat color (Senda et al. 2002). The $I$ gene and the A2D8 linked markers are very strongly associated with SCN traits and recombination events are rare (Weiseman et al. 1992; Meksem et al. 2001). However, recombination events suggest the rhgl locus lies between A2D8 and BLT65, a $142 \mathrm{kbp}$ region encompassing genes encoding a subtilisin-like protein and a transporter like protein (Campbell et al. 2009). 
Additional QTL identified in 'PI 437654', 'Hartwig' and PI88788 indicated another three QTL underlie resistance to HG Types 1.3- and 1.2.5- which may be located on linkage groups (LG) J (Rhg5), D2 (Rhg2), and C1 (Rhg3; Webb et al. 1995; 1996; Schuster et al. 2001; Glover et al. 2004). Four different loci (on LGs A1, B1, F and G) underlying resistance to SCN were detected in PI437654 using inbred nematodes (Vierling et al. 1996). More recently the number of loci underlying resistance to SCN mapped in different populations derived from PI437654 with a range of HG Types has risen from those nine to ten (LG I), if only additive loci were counted, but eighteen (all LGs except B1 and D1b) if epistasis among loci was measured (Wu et al. 2009). Possibly the PI437654 genome encodes many redundant loci for resistance to SCN that may be activated by crosses to different susceptible cultivars or by challenge with different $\mathrm{HG}$ Types.

A RIL population was developed from the cultivar Hartwig to assist with mapping agronomic traits including SCN, SDS, seed yield and yield components (Prabhu et al. 1999; Yuan et al. 2002; Kazi, 2005; et al 2006; et al 2007). Since cv. 'Hartwig' is the product of 3 backcrosses of Forrest with 'PI437654' as the SCN resistance donor (Anand 1992) some loci underlying resistance to SCN might be introgressed and others not (Schuster et al. 2001). Therefore, confirmation of QTL in near isogeneic lines (NILs) will be important. NILs derived from residual heterozygosity in RILs at the F5 are widely used for this purpose (Njiti et al. 1998; Meksem et al. 1999; Triwtayakorn et al. 2005; Ruben et al. 2006; Farias-Neto et al. 2007). The use of NILs separates the QTLs effects from one another and other interacting loci (Wu et al. 2009). 
The research described here sought to confirm previously reported QTL (Webb et al. 1995; 1996; Vierling et al. 1996; Schuster et al. 2001; Wu et al. 2009) or identify new QTL that underlie resistance to SCN. A 'Flyer' by Hartwig derived recombinant inbred line (RIL) population challenged with three different HG Types (0, 1.3-, and 1.2.5-) was used. A new QTL was identified in RILs, confirmed using NILs and the confirmed QTL assigned the designation cqSCN-005. 


\section{MATERIALS AND METHODS}

\section{Plant Material}

Seeds were developed by the Genetics and Biotechnology program at Southern Illinois University at Carbondale at the Agronomy Research Center (ARC) from 19932001. For this research seeds were obtained from the collections of Dr. D.A. Lightfoot in the ARC seed store at SIUC in 2002. The RILs derived from a cross between two soybean cultivars, susceptible Flyer and resistant Hartwig $(\mathrm{F} \times \mathrm{H}, \mathrm{n}=92 ; \mathrm{FH} 92)$ and the population was released in 2006 ( Kazi et al. 2007). Flyer was originally released for high seed yield combined with a wide range of fungal resistance loci (McBlain et al. 1990).

All RILs in FH92 were selected for agronomic type. Fifty of the RILs were selected for the presence of approximately equal numbers of alleles at Satt038 (LG G) and BLT65 (LG A2; Prabhu et al. 1999). The selection for agronomic type tends to select against SCN resistant lines (Yuan et al. 2002; Brucker et al. 2005; Kopisch-Obuch et al. 2005). Also the SCN resistance alleles at rhgl must be in phase with an unlinked modifier locus for gametes or seed to be viable (Webb et al., 1995; 1996; Afzal et al. 2008; 2009). Therefore, marker selection for RILs with resistance alleles at rhgl and Rhg4 was necessary to avoid a skewed and biased population formed by these unintentional selections.

\section{SCN female index (FI) determination:}

The SCN assays used previously described methods (Prabhu et al. 1999; Arelli et al. 2000; Yuan et al. 2002) for HG Types 0 and 1.3-, but with minor modifications for 
HG Type 1.2.5-. For the $\mathrm{F}_{5: 11}$ FxH RIL (n=94) population SCN HG Types 0 and 1.3- used five and three single-plant replications respectively. For HG Type 0 tests the cultivar 'Hutcheson' was used as the susceptible control while 'Peking' (female index; FI 2\%), 'PI 88788' (FI 3\%), 'PI 90763' (FI 1\%) and 'Pickett' (FI 3\%) were used as the standard differentials to determine race classification. For HG Type 1.3- tests the cultivar 'Hutcheson' was used as the susceptible control while Peking (FI 98\%), PI 88788 (FI 3\%) and PI 90763 (FI 101\%) and Pickett (FI 68\%) were used as the standard differentials to determine the race classification (HG Type). Assays were carried out at the University of Missouri by P. Arelli using near homogeneous nematode cultures. The development of the near homogenous nematode cultures was described in Qiu et al. (1999).

The HG Type 1.2.5-(race 2) assays were replicated at SIUC by Dr. Bond's staff and at the University of Missouri by Dr. Arelli's staff. The RIL experiments each used two single plant replications (four plants in total), while the NIL experiments used six single-plant replications (twelve plants in total). The cultivars 'Lee 74', 'Essex' and 'Hutcheson' were used as susceptible controls (Niblack et al. 2003). The differentials or indicator lines were 'PI54840' (FI 101\%), PI 88788 (FI 24\%), PI90763 (FI 1\%), PI437654 (FI 0\%), 'PI 209332' (FI 61\%), 'PI89772' (FI 2\%) 'PI548316' (FI 38\%) and 'PI548402' (FI 35\%) in the first experimental repeat. In the second repeat the FIs were $19 \%, 48 \%, 0 \%, 0 \%, 63 \%, 0 \%$ and $39 \%$. Therefore the standard differentials showed this HG Type to be 1.2.5- (Niblack et al. 2003) corresponding to race 2 (Riggs and Schmitt, 1988).

For inoculation, seeds were surface sterilized with $5 \%(\mathrm{v} / \mathrm{v})$ bleach for $2 \mathrm{~min}$, rinsed three times with distilled water and placed on autoclaved filter paper in Petri 
dishes (Arelli et al. 2000). These dishes were incubated in the dark at $20{ }^{0} \mathrm{C}$ for 48 hours. Seeds were planted separately in plastic Micropots ${ }^{\mathrm{TM}}$ (Hummert International, Earth City, MO), then immersed in a $27^{0} \mathrm{C}$ water bath. Seven days after seedling emergence, each pot was inoculated with 1-ml of crushed cysts from a homogenous isolate of H. glycines in distilled water $(2,000 \pm 25$ eggs per $\mathrm{ml})$.

Thirty-eight days after inoculation, individual plants were uprooted, and the cysts were collected by washing the roots with pressurized water over wire mesh sieves. The leaves from the top of these plants were stored at $-70^{\circ} \mathrm{C}$. The total numbers of cysts were counted using a Nikon (Melville, NY) dissecting scope (Model SMZ645) at 10x magnification. The mean number of cysts from two to six single-plant replications (for each line) and the susceptible checks were determined. The FI, previously called Index of Parasitism (IP), Schmitt and Shannon, (1992) was calculated as the number of cysts or females on the evaluated genotype divided by the mean number of cysts or females present on the susceptible cultivar roots $\mathrm{x} 100$.

\section{DNA Marker Analysis}

DNA was extracted and used for microsatellite amplifications as in Yuan et al. (2002) with modifications described in Kazi et al. (2008). Briefly, BARC-Satt markers were chosen at $10 \mathrm{cM}$ intervals from the soybean genetic map (Song et al. 2004). In addition, SIUC-BES-SSR markers from build 2 MTP BES clones (Shultz et al. 2006ab; 2007) were chosen at $10,000 \mathrm{kbp}$ intervals within the soybean physical map (Shultz et al. 2006ab; 2007) and in the regions of rhgl (SIUC-TMD1) and Rhg4 (SIUC-B100B10b). The six markers added compared to Kazi et al. (2008) were Satt038_1, Satt038_2 on LG 
G near rhg1 and BLT65, H100B10b, H100B10a and A2D8 on LG A2 encompassing Rhg4 (Supplemental Table 1)

\section{Trait Heritability and Correlation Estimations}

Heritability $\left(\mathrm{h}^{2}\right)$ estimates, a ratio of genotypic variation over phenotypic variation of SCN FI\%, were calculated using variance components obtained through ANOVA as described in Fehr (1987). Due to the low frequency of heterozygosity at the $F_{5: 11}$, the genetic variance is almost entirely an additive and additive $\mathrm{x}$ additive interaction. Therefore, heritability was considered narrow.

All correlations were calculated using the PROC CORR function of SAS (SAS Institute, Cary, NC) using line mean data. The SDS data were described in detail in Kazi et al. (2007) as mean disease index (leaf scorch; two locations, one year) and mean infection severity (root infection; four locations two years). The mean seed yield data was described in detail in Yuan et al. (2002) and was from four environments and 2 years. The SCN FI data were the mean number of cysts from two to six single-plant replications (for each line) and two experiments.

\section{Construction of the Genetic Linkage Map}

The linkage map was created using MAPMAKER/EXP 3.0 (Lander et al. 1987) as described by Kazi et al. (2008). Briefly, map distances were calculated in Haldane units; heterogenous lines were excluded; the RI-self genetic model was used. The LOD for grouping markers was set at 3.0 with a maximum distance of $50 \mathrm{cM}$ and computed with error detection. Most markers were anchored on the LGs on the basis of the locations 
expected from the composite map (Song et al. 2004). Conflicts among the positions of linked markers in $\mathrm{FxH}$ were resolved in favor of experimental evidence if there was evidence for the existence of paralogs of marker amplicons. Paralogs were inferred when the maps generated by Mapmaker had linkage probabilities above LOD 3.0 that disagreed with the composite map. Most markers have homeologous loci in soybean (Shultz et al. 2006a; Saini et al 2008).

\section{Construction of QTL Maps}

\section{A. Single Point Analysis}

For line mean comparisons, the data were subjected to analysis of variance (ANOVA; SAS Institute Inc., Cary, NY), with mean separation by LSD as described by Kazi et al (2008) for RILs and Njiti et al. (1998) for NILs. Markers were compared with SCN FI measures by the F-test of ANOVA. Heterogeneous lines were excluded from analysis (numbers of lines ranged from 3 to 15 with a mean of 8.3 per marker).

A significant difference $(P<0.005)$ was considered to be a preliminary indication of an association between a marker and a QTL for the trait in question. A value of $\mathrm{P} \leq$ 0.0005 was suggested by an approximate Bonferroni correction $(\mathrm{P}<0.05 / 100)$ for the set of about 100 independent (unlinked or $>10 \mathrm{cM}$ apart) DNA markers (from the 144 mapped). However, at genomic regions where markers were sparse and gaps between adjacent markers were greater than $10 \mathrm{cM}$ associations in the range $0.005>\mathrm{P}>0.0005$ were considered significant associations. If the interval was large or was flanking a single marker the uncorrected $\mathrm{P}$ value of $<0.05$ was accepted. Precedents with first-pass mapping of other quantitative traits (Hnetkovsky et al. 1996, Chang et al. 1997; Njiti et 
al. 2002) have shown these criteria to be valid during subsequent saturation mapping of intervals inferred at marginal P values (Njiti et al. 1998; Meksem et al. 2001; Yuan et al. 2002; Triwitaykorn et al. 2005; Ruben et al. 2006).

B. Interval Maps of QTL

Maps of all linked markers and trait data were simultaneously analyzed with Mapmaker/QTL 1.1 using the $\mathrm{F}_{2}$-backcross genetic model for trait segregation (Chang et al. 1997, Njiti et al. 2002; Kazi et al. 2008). Putative QTL were inferred when LOD scores exceeded 2.0 at some point in an interval. LOD 2.0 was empirically determined to be equivalent (but not equal) to a single marker $\mathrm{P}<0.005$. The position of each QTL was inferred from LOD peaks at individual loci detected by maximum likelihood tests at positions every $2 \mathrm{cM}$ between adjacent linked markers.

C. Composite Interval Maps of QTL

For more accurate location of QTL among sets of linked markers, the composite interval map (CIM) function of WinQTL Cartographer (version 2.5) was used (Jansen and Stam 1994; Basten et al. 2001). Following Kazi et al. (2008) a walk speed of $2 \mathrm{cM}$ and the forward regression method were selected. QTL were inferred when LOD score peaks exceeded 2.0 for the traits studied, considering a $\mathrm{P}<0.05$ corrected for the use of about 100 independent markers. To confirm linkage, experiment-wise threshold was calculated from 1,000 permutations of each genotype marker against the phenotype in the population. 


\section{RESULTS}

\section{Polymorphism and Linkage.}

The linkage map used is described in Kazi et al. (2008) with six additional markers. Briefly, one hundred and fifty markers were found to be polymorphic within a Flyer x Hartwig (FxH) RIL population (104 of 250 BARC-SSRs, 40 of 143 BES-SSRs and 3 of 3 SCARs tested). There were 3-10 markers per linkage group and distance between markers was 10-25 cM except for linkage groups, G (Meksem et al. 1999) and K that were tested with additional markers due QTL detections reported previously (Yuan et al. 2002, Kazi et al. 2008). Sixty one markers formed 17 linkage groups encompassing $534 \mathrm{cM}$. The map has large gaps because the RIL population was derived from a cross between cultivars with a high coefficient of common ancestry $(\sim 0.25)$ from a very small germplasm base, north American adapted soybean cultivars. However, assuming $10 \mathrm{cM}$ as a distance for QTL detection, the groups formed plus the 81 unlinked markers would allow the detection of QTL over 2,494 cM (Supplemental Figure 1). The recombination distances and orders of markers in linkage groups (with 2-3 exceptions) and genome size (2,512 cM) agreed with those reported (Song et al. 2004).

\section{Variation of resistance within FxH RILs to three races of SCN.}

The means and standard errors of phenotypic variation of FI among the two parents and RILs within all three HG Types were relatively constant with minor variations (Table 1) indicating that the SCN bioassays were useful for further analysis. Transgressive segregation was observed in HG Type 0 (race 3) FI scores but not in HG Type 1.2.5(race 2) and HG Type 1.3- (race 14; Fig 1A, 1B, 1C). The RIL population mean was 
intermediate to the two parents for each HG Type (Table 1). Narrow sense heritability estimates for SCN FI had relatively high values (0.95 for HG Type $0,0.96$ for HG Type 1.2.5-, and 0.86 for HG Type 1.3-; Table 1).

Overall responses within the RILs to the HG types produced continuous distributions (Fig 1A, 1B and 1C), including HG Type 1.2.5- $(\mathrm{n}=14)$ for which only 14 lines were selected as R to HG Type 0 or HG Type 1.3-. The Shapiro-Wilks Test (W test), showed a normal distribution $(\mathrm{W}=0.95 ; P=0.73$; Fig. 1A). The distribution was skewed (-0.58) towards the low female index (Hartwig). A negative kurtosis (-0.59) reflected a platykurtic distribution caused by a few extreme scores (two resistant RILs FI<10; FxH35 and FxH93). There were no significant transgressive segregants.

For resistance to HG Type 0, often a bigeneic trait (Meksem et al. 2001), the distribution was not normal ( $\mathrm{W}=0.95 ; P=0.001$; Fig. $1 \mathrm{~B})$. The distribution was skewed $(-0.44)$ towards the low female index (Hartwig). The distribution was negatively kurtotic (-0.72). There were about 18 lines with a mean FI higher than Flyer that are transgressive segregants (Fig. 1B). There were 14 resistant lines $(\mathrm{FI}<10)$ and another 14 moderately resistant lines $(10>\mathrm{FI}>30)$. Only five lines were equal in resistance to Hartwig $(\mathrm{FI}<$ 1.0) but non were significant transgressive segregants.

HG Type 1.3- FI distribution was not normal as $\mathrm{W}=0.87(P=0.0001$; Fig $1 \mathrm{C})$ and was skewed (1.5) towards the high female index (Flyer). There was a significant positive kurtosis (2.75) reflected a leptokurtic distribution. No significant transgressive segregants were observed. There were only 4 resistant lines $(\mathrm{FI}<10)$, no moderately resistant lines $(10>$ FI $>30)$ and only 11 moderately susceptible lines $(30<$ FI <60). None of the four resistant lines were equal in resistance to Hartwig $(\mathrm{FI}<1.0)$. 


\section{Correlations among SCN, SDS and Seed Yield Traits:}

Correlations among SDS (DX, ISR6, ISR8), SCN (FI 0, FI 1.3-) and seed yield (Mg/Ha) were made toexpand on the reports of Yuan et al. (2002) and Kazi et al. (2008). Resistance to SCN HG Type 0 was negatively correlated with seed yield $\left(R^{2}=0.69\right)$. Resistance to HG Type 1.3- was also negatively correlated with seed yield but more weakly $\left(R^{2}=0.26\right)$. Positive correlations were observed when comparing resistance to SCN HG Type 0 with the resistance to infection (IS) of roots by Fusarium virguliforme (the pathogen that causes SDS) at growth stages R6 $\left(R^{2}=0.71\right)$ and $\mathrm{R} 8\left(R^{2}=0.75\right)$ whereas SDS DX was weakly correlated $(R=0.31)$. However, the correlation between infection severity with HG Type 1.3- FI showed a negative correlation at both R6 $\left(R^{2}=\right.$ $0.27)$ and IS-R8 $\left(R^{2}=0.63\right)$. Correlations inferred the relationships between loci underlying responses to SCN, SDS and seed yield would include linkages in attraction and repulsion phase at separate loci.

\section{Identification of QTL underlying resistance to SCN FI HG Types 0 (race 3) and 1.3- (race 14) in the RIL population}

The major genomic region associated with variation in resistance to HG Type 0 and HG Type 1.3- was found on linkage group G, the expected position for rhgl (Table 2). The marker Satt309 was the marker most strongly associated with resistance to HG Type $0\left(P=0.0001, \mathrm{R}^{2}=22.3 \%\right)$. The beneficial allele was derived from Hartwig $(\mathrm{H})$ not Flyer $(\mathrm{F}=64.3 \pm 4.1, \mathrm{H}=20.8 \pm 6.8)$. The TMD1 marker from the intron in the RLK gene at $r h g 1$ was also strongly associated $\left(P=0.008, \mathrm{R}^{2}=11 \%\right)$ and closely linked $(3.3$ $\mathrm{cM})$. The beneficial TMD1 region was also derived from Hartwig $(\mathrm{F}=60.8 \pm 4.2, \mathrm{H}=$ 
$32.6 \pm 9.1)$. The Satt309 to TMD1 interval had a peak-LOD score of 3.9 and explained 21 $\%$ of the total variation in the HG Type 0 FI. Markers Satt275 $\left(2.2 \mathrm{cM} ; P=0.002, \mathrm{R}^{2}=\right.$ $13.7 \%)$ and Satt163 (3.2 cM; $\left.P=0.005, \mathrm{R}^{2}=14 \%\right)$ located to the telomeric side of TMD1 were less strongly associated with resistance to HG Type 0.

Markers to the centromeric side of Satt309 were also strongly associated with resistance to HG Type 0. Satt610 $\left(P=0.001, \mathrm{R}^{2}=17 \%\right), 15.5 \mathrm{cM}$ from Satt309, had the beneficial allele from Hartwig $(\mathrm{F}=66.5 \pm 5.6, \mathrm{H}=34.2 \pm 6.5$; Table 2$)$. A high peakLOD score of 4.85 underlay $27 \%$ of the trait variation. However, selection against inheriting Hartwig alleles at $r h g 1$ was observed for Satt 309, TMD1 Satt163 and Satt275. In contrast Satt038_2 and Satt610 showed segregation ratios that were not significantly skewed away from 1:1 ratios among the RILs. Consequently Satt038_2 was placed between Satt309 and Satt610 (Figure 3; Kazi et al. 2008), an unusual position for Satt038_1 on the composite map (Song et al. 2004). Therefore, interacting loci may be involved and caution should be exercised in changing the position of the QTL at rhg 1 from the TMD1 to Satt309 interval expected (Ruben et al. 2006).

Markers linked to Rhg4 on LG A2 (BARC-BLT65, SIUC-B100B10b and SIUCA2D8) were significantly associated with resistance to HG Type 0 . The marker most strongly associated was A2D8 $\left(\mathrm{P}<0.001, \mathrm{R}^{2}=19 \%\right)$ and the beneficial allele was again from Hartwig $(\mathrm{F}=51.2 \pm 4.5, \mathrm{H}=25.0 \pm 2.5)$. The association was not detected by Prabhu et al. (1999) since only 50 lines were phenotyped at that time and only BLT65 was used. Here, 92 lines and the parents were tested with HG Type 0 and A2D8, a marker more closely linked to Rhg4 $(0.25 \mathrm{cM})$ than BLT65 (about $1.25 \mathrm{cM})$ was used (Meksem et al. 2001). 
For HG Type 1.3-, the Satt309 region of LG G was significantly associated with resistance $\left(P=0.001, \mathrm{R}^{2}=16.6 \%\right.$; Table 3$)$, with the beneficial allele originating from Hartwig $(\mathrm{F}=81.2 \pm 2.30, \mathrm{H}=69.1 \pm 5.76)$. The linked $(3.2 \mathrm{cM})$ marker Satt163 $(P=$ $0.001, \mathrm{R}^{2}=6 \%$ ) was also associated. The interval had a peak-LOD score of 2.87 and explained about $14.6 \%$ of trait variation in the FxH RIL population. Markers linked to Rhg4 on LG A2 (BLT65, B100B10b and A2D8) were weakly associated with resistance to HG Type $1.3-(0.05<\mathrm{P}<0.01)$.

For HG Type 1.2.5-, as sub-set of RILs $(n=14)$ was used for ANOVA, the lines that were HG Type 0 resistant. Analysis indicated that the region of LG G from Hartwig identified by Satt309 was significantly associated with resistance $\left(P=0.001, \mathrm{R}^{2}=24 \%\right)$ in the RIL sub-population. Markers linked to Rhg4 on LG A2 (BLT65, B100B10 and A2D8) were not significantly associated with resistance to HG Type 1.2.5- in this small sub-set (data not shown). However, another marker Satt543 on LG D2 was significantly

associated with resistance to $\mathrm{HG}$ Type $1.2 .5-\left(P=0.005, \mathrm{R}^{2}=12 \%\right)$. The possible associations with resistance to HG Type 1.2.5- were tested in NIL populations to attempt QTL confirmation.

\section{Confirmation of loci associated with resistance to SCN FI HG Type 1.2.5- (race 2) in a NIL population}

Because there were only two RILs resistant to HG Type 1.2.5- , QTL analysis in the 14 lines most resistant to HG Type 0 was followed by analysis in NILs derived from RILs. Judged by single plant responses, RILs found to be heterogeneous with one of the 
QTL associated markers or segregating for resistance to SCN were selected for further NIL analysis (Kazi 2005).

A near-isogenic line (NIL) population, derived from RIL FxH19 was used to confirm the location of the locus underlying the SCN HG Type 1.2.5-resistance. Ten NILs were extracted and six plants per NIL were used for FI determination. The frequency distribution of FxH19-derived NIL population appeared continuous and positively kurtotic (data not shown; see Kazi, 2005). The female index ranged from 12 to $>114$ and was skewed toward a high female index. Heritability was calculated at $91 \%$. Line 19-8 was the only fully resistant line, two lines were moderately susceptible $(\mathrm{FI}<60)$, and seven NILs were susceptible. Within intermediate lines $(12>\mathrm{FI}<100)$ there were some individual plants with FI $<10$, suggesting genetic segregation within the NILs or line contamination or some escapes from infection.

The RIL FxH19 and derived F5:7 derived NIL FH19-1 to 20 all carried the Hartwig allele at rhgl (TMD1) and Rhg4 (Satt089) as judged by DNA markers. The NILs FH19-1 to 10 was expected to have about $6.25 \%$ of the genome segregating, but did not segregate for 140 of the 144 markers tested suggesting only $3 \%$ of loci were segregating. However, the four markers that were heterogeneous in FxH RIL 19 segregated in the derived near isogenic line population. Table 4 shows that mean FI was significantly lower $\left(P<0.046, R^{2}=19 \%\right)$ for genotypes carrying the Hartwig allele for Satt543 (LG D2; 88.02 cM). However, Hartwig alleles at Satt594 (LG G; 52.93 cM), Satt237 (LG N; $74.98 \mathrm{cM}$ ) and Satt530 (LG N; 32.84) were not associated with different SCN FI means. The most resistant NIL 19-8 showed a single plant that was susceptible 
(FI 60\%) and five completely resistant plants (Figure 2 B). The susceptible plant had the Flyer allele at Satt543 suggesting the NIL 19-8 was still segregating at this locus.

A second small NIL population $(\mathrm{n}=10)$ derived from FxH33 showed segregation at markers linked to rhgl (cqSCN-001) on LG G. There was association between the HG Type 0, 1.3- and 1.2.5- traits and those markers (data not shown; Kazi, 2005). This was an expected result that validated the usefulness of the small NIL populations for QTL confirmation.

\section{The seed yield QTL on LG D2}

The region on LG D2 (Figure 3) identified by the microsatellite marker Satt514 was significantly ( $P=0.0006, R^{2}=7.4 \%$ ) associated with seed yield in the RILs of FH92. The QTL was detected in the across environment grand mean yield, from 1998-1999, in the complete population of 92 RILs grown at four locations (two row plots, three replications). The beneficial allele was from Hartwig $\left(3.00 \pm 0.04 \mathrm{Mg}^{-1} \mathrm{a}^{-1}\right)$ not Flyer $\left(2.77 \pm 0.05 \mathrm{Mg} \mathrm{ha}^{-1}\right)$. The adjacent marker was Satt488. The interval between these two markers spanned a genetic distance of about $3.5 \mathrm{cM}$. The yield QTL had peak Loglikelihood (LOD) of 2.57 and explained about $13.3 \%$ of total variation in seed yield $(0.42$ $\left.\pm 0.04 \mathrm{Mg} \mathrm{ha}^{-1}\right)$.

\section{Discussion}

The correlations among SCN SDS and seed yield traits might be explained by the different linkage phases among separate loci for seed yield and resistances to SCN and SDS found on LGs G and D2 reported here (Table 1-3; Figure 3). On LG G the alleles 
underlying resistance to SCN and SDS are linked to a seed yield depression allele. On linkage group D2 the reverse is true, the SCN resistance allele is linked to a seed yield increase allele but an allele underlying SDS susceptibility. Therefore, progress can be made in soybean breeding by identifying recombination events in both these regions with new linkage relationships.

Resistance to SCN Hg Type 1.3- was shown to be a dependent trait requiring resistance alleles at both HG Type 0 loci ( $r h g 1$, Rhg4) and one extra locus. The linkages observed could also explain how resistance to SCN Hg Type 0 is positively correlated with resistance to SDS whereas resistance to SCN Hg Type 1.3.5is negative correlated with SDS both in this population (Kazi et al. 2008) and across diverse germplasm (Gibson et al. 1994). However, sample size may have a large effect on the correlations reported here. With SCN data the correlations were strongly affected by; the biphasic nature of the trait distributions (Figure 1); the small number of resistant lines (14 for HgType 0; 4 for HgType 1.3.- and 2 for HgType 1.2.5); the large number of susceptible lines; and scarcity of lines with intermediate FI scores.

The major effect of $r h g l$ on resistance to all HG Types (Webb et al. 1996; Concibido et al. 1996; 2004) was confirmed (Table 5). The effect of Rhg4 on resistance to $\mathrm{Hg}$ type 0 and $\mathrm{Hg}$ Type 1.3- was also confirmed. An additional locus for resistance to Hg Type 1.3- was inferred from the fact that only 4 resistant lines were identified from 14 resistant to $\mathrm{HgType}$ 0, but the marker association with that locus (expected on $\mathrm{LgCl}$; Webb et al. 1995) was not made to date (Table 5). Only two lines were resistant to HgType 1.2.5- so that as well as the locus on $\mathrm{LgD} 2$ another (on $\mathrm{Lg} \mathrm{J}$ ) may underlie 
resistance (Table 5; Webb et al. 1995). Some genomic regions were not polymorphic (Supplemental Figure 1). Only $40 \%$ of BARC-Satt markers and 30\% of BES-SSR markers were polymorphic. This lack of polymorphism in large regions of the genome occurs because the cross was between cultivars with a high coefficient of common ancestory $(\sim 0.25)$ from a very small germplasm based, north American adapted soybean cultivars. There are large regions of the genome expected to be monomorphic between Hartwig and Flyer. However, the regions underlying resistance to SCN would have to be polymorphic. Some of the polymorphic regions underlying resistance to SCN may be quite small because Hartwig is the product of a series of backcrosses (Forrest ${ }^{3}$ xPI437654; Anand et al 1992). Consequently, much greater marker density may be needed to detect the additional loci.

The loci intervals seemed to shift depending on the HG Type used even though the same set of RILs was included in each analysis (Figure 3). Among possible causes of the shifts were; the subset of resistant lines was different in each case; sampling errors caused by experimental variations in SCN FI; the action of multiple, linked genes (Ruben et al. 2006; Afzal et al 2009); un-intentional selections against resistance alleles at the rhgl locus (Prabhu et al. 1999); or interactions among rhg-loci (Webb et al. 1995; 1996; Wu et al 2009). Unintentional selection did have an effect as only eighteen RILs had the alleles associated with resistance at both markers most closely encompassing rhg 1 (Satt 309 and TMD1; Ruben et al; 2006; Afzal et al. 2009). Therefore, the small sample size of RILs resistant to SCN caused by natural or unintentional selection appeared most likely to explain the subtle shifts in the rhgl position with each HG Type. One line with a 
recombination event between rhgl and the nearest marker or with an unusual phenotypic score would be sufficient to cause such a shift.

The Rhg-genes clearly interact as no single gene is sufficient for resistance. In addition the loci have paralogs. The set of genes identified within the rhgl locus (Ruben et al. 2006) have a syntenic set of paralogs at another genomic location on LG B1 (Afzal and Lightfoot 2007; http://www.phytozome.net/cgi-bin/gbrowse/soybean/; Afzal et al. 2008; 2009). Interactions among rhg-loci are common phenomena (Webb et al 1996; Wu et al 2009; Afzal et al 2009). However, the interacting loci on LG M inherited in phase with rhgl (Webb et al. 1995; 1996) did not appear to segregate in FH92 since no marker on LG M was polymorphic among the 20 tested. The equivalent locus discovered in ExF in the middle of LG G was not co-inherited with rhgl either. Therefore, the known modifiers of rhgl action could not be implicated in the shift in apparent location of the loci.

On LG D2 the locus underlying resistance to HG Type 1.2.5- might be the same allele as that underlying race 1.3- in the population derived from Hartwig by BR-9231983 (Schuster et al. 2001) or simply linked to it. However, it was not associated with resistance to $\mathrm{Hg}$ Type 1.3- in the experiments reported here. The locus detected here was confirmed to underlie resistance to $\mathrm{Hg}$ Type 1.2.5- in a small NIL population and therefore represented a confirmed QTL. The designation cqSCN-005 was assigned by the Soybean Genetics Committee in 2008 (http://www.soygenetics.org/committee.php). The Hartwig allele at the region seemed to increase seed yield by $0.3 \mathrm{Mg} \mathrm{ha}^{-1}$ (either by resistance to low SCN infestations or linkage to a second locus) but caused greater susceptibility to root infection (IS by 15\%) and leaf scorch (by 7\%; Kazi, 2005; Kazi et 
al. 2008). Therefore, the D2 locus should be targeted for fine mapping, genomic analyses, and marker selection of new recombination events that improve all three agronomic traits.

The molecular basis of the interactions among loci; within resistance to SCN (Webb et al. 1995; 1996; Wu et al. 2009); among diseases (Kazi et al. 2007); and with agronomic traits (Yuan et al. 2002) is not clear. A comparison of Williams, Forrest, Hartwig and PI437654 DNA sequences in both the G and D2 regions associated with resistance to SCN (Lightfoot, 2008; Afzal et al. 2009; Campbell et al. 2009) might show the molecular mechanisms underlying these interactions, correlations and phenomena.

\section{Acknowledgements}

This research was funded over the past 11 years in part by grants from the NSF 9872635 , ISA 95-122-04; 98-122-02 and 02-127-03 and USB 2228-6228. The integrated genetic and physical map was based upon work supported by the National Science Foundation under Grant No. 9872635. Any opinions, findings, and conclusions or recommendations expressed in this material were those of the author(s) and do not necessarily reflect the views of the National Science Foundation or United States Department of Agriculture. The continued support of SIUC, College of Agriculture and Office of the Vice Chancellor for Research to SK, JA and DAL was appreciated. The authors thank Dr.s P. Gibson, O. Myers Jr. and M. Schmidt for assistance with germplasm development and maintenance from 1991-2000. We thank the "Soybean Genome Project", at DoE Joint Genome Institute" for release of the WGS reads, scaffolds and genome sequence. 


\section{References}

Afzal AJ, Lightfoot DA (2007) Soybean disease resistance protein RHG1-LRR domain expressed, purified and refolded from Escherichia coli inclusion bodies: preparation for a functional analysis. Protein Expr Purif 53:346-55

Afzal AJ, Saini N, Srour A, Lightfoot DA (2008) The multigeneic rhgl locus: a model for the effects on root development, nematode resistance and recombination suppression. Nature Precedings hdl:10101/npre.2008.2726.1 (online).

Afzal AJ, Saini N, Srour A, Lightfoot DA (2009) The multigeneic rhgl locus complete sequence identifies alleles of three genes underlying root development, nematode resistance and recombination suppression. Nature Genetics (in review)

Anand SC (1992) Registration of 'Hartwig' soybean. Crop Sci 32:1060-1070

Arelli PR, Sleper DA, Yue P, Wilcox JA (2000) Soybean reaction to races 1 and 2 of Heterodera glycines. Crop Sci 40:824-826.

Basten CJ, Weir BS, Zeng Z (2001) QTL Cartographer Version 2.0. Raleigh, NC: Department of Statistics, North Carolina State University, USA

Brucker E, Niblack T, Kopisch-Obuch FJ, Diers BW (2005) The effect of $r h g 1$ on reproduction of Heterodera glycines in the field and greenhouse and associated effects on agronomic traits. Crop Sci 45:1721-1727

Campbell N, Warner AL, Lightfoot DA, Matthews BF, Knap HT (2009) Duplication of a chromosomal region from linkage group A2 involved in cyst nematode resistance in soybean. Mol Gen Genet (in press). 
Chang SJC, Doubler TW, Kilo V, Suttner RJ, Klein III JH, Schmidt ME, Gibson PT Lightfoot DA (1997) Association of field resistance to soybean sudden death syndrome (SDS) and cyst nematode (SCN). Crop Sci 37:965-971

Concibido VC, Lange DA, Denny RL, Orf JH, Young ND (1997) Genome mapping of soybean cyst nematode resistance genes in 'Peking', PI 90763, and PI 88788 using DNA markers. Crop Sci 37:258-264.

Concibido VC, Diers BW, Arelli PR (2004) A decade of QTL mapping for cyst nematode resistance in soybean. Crop Sci 44:1121-1131

Davis EL, Hussey RS, Baum TJ ( 2004) Getting to the roots of parasitism by nematodes. Trends Parasitol 20:134-41.

Fehr,W (1987) Principals of cultivar development. Vol. 1: Theory and Techniques. McMillan, New York, USA

Gibson PT, Shenaut MA, Njiti VN, Suttner RJ, Myers Jr O (1994) Soybean varietal response to sudden death syndrome. p. 436-446. In D. Wilkinson (ed.) Proc. Twenty-fourth Soybean Seed Res. Conf., Chicago, IL. 6-7 Dec. 1994. Am Seed Trade Assoc Washington DC.

Glover KD, Wang D, Arelli PR, Carlson SR, Cianzio SR, Diers BW (2004). Near Isogenic lines confirm a soybean cyst nematode resistance gene from PI88788 on linkage group J. Crop Sci 44:936-941.

Hussey RS, Grundler FMW (1998) Nematode parasitism of plants. In: Perry RN, Wright DJ (eds) The physiology and biochemistry of free-living and plant parasitic nematodes. CAB International, Wallingford, pp 213-243 
Hnetkovsky N, Chang SJC, Doubler TW, Gibson PT, Lightfoot DA (1996) Genetic mapping of loci underlying field resistance to soybean sudden death syndrome (SDS). Crop Sci 36:393-400

Iqbal M.J., Ahsan R, Afzal AJ, Jamai A, MeksemK, El- Shemy HA, Lightfoot DA (2009) Multigeneic QTL: The laccase encoded within the soybean $R f s 2 / r h g 1$ locus inferred to underlie part of the dual resistance to cyst nematode and sudden death syndrome. Curr. Issues Mol. Biol. i11: 11-19

Jansen RC, Stam P (1994) High resolution of quantitative traits into multiple loci via interval mapping. Genetics 136: 1447-1455

Kazi S (2005) Minimum tile derive microsatellite markers improve the physical map of the soybean genome and the Flyer by Hartwig genetic map at $R h g, R f s$ and yield loci. MS Thesis SIUC Carbondale IL, USA, pp 212

Kazi S, Njiti VN, Doubler TW, Yuan J, Iqbal MJ, Cianzio S, Lightfoot DA (2007) Registration of the Flyer by Hartwig recombinant inbred line mapping population. J Plant Regis 1: 175-178

Kazi S., Shultz J, Bashir, R., Afzal J, Njiti V, Lightfoot DA (2008) Separate loci underlie resistance to soybean sudden death syndrome in 'Hartwig' by 'Flyer'. Theoretical and Applied Genetics 116: 967-977

Kopisch-Obuch FJ, McBroom KL, Diers BW (2005) Association between SCN resistance loci and yield in soybean. Crop Sci 45:956-965

Lander E, Green P, Abrahamson J, Barlow A, Daley M, Lincoln S, Newburg L (1987) MAPMAKER:An interactive computer package for constructing primary genetic linkage maps of experimental and natural populations. Genomics 1:174-181 
Lightfoot DA (2008) Soybean genomics: Developments through the use of cultivar Forrest. Internat J of Plant Genom 2008:1-22. doi:10.1155/2008/793158

McBlain BA, Fioritto RJ, St Martin SK, Calip-DuBois A, Schmitthenner AF, Cooper RL, Martin RJ (1990) Registration of 'Flyer' soybean. Crop Sci 30:425

Meksem K, Doubler TW, Chancharoenchai K, Njiti VN, Chang SJC, Rao-Arelli AP, Cregan PE, Gray LE, Gibson PT, Lightfoot DA (1999) Clustering among loci underlying soybean resistance to Fusarium solani, SDS and SCN in near-isogenic lines. Theor Appl Genet 99:1131-1142

Meksem K, Pantazopoulos P, Njiti VN, Hyten DL, Arelli PR, Lightfoot DA (2001) 'Forrest' resistance to the soybean cyst nematode is bigenic: saturation mapping of the rhgl and Rhg4 loci. Theoretical and Applied Genetics 103:710-717.

Niblack, TL, Noel, GR, Lambert, KL (2003) The Illinois SCN type test: Practical application of the HG Type classification system. J Nematol 35: 355-345

Njiti VN, Doubler TW, Suttner RJ, Gray LE, Gibson PT, Lightfoot DA (1998) Resistance to soybean sudden death syndrome and root colonization by Fusarium solani f. sp. glycines in near-isogeneic lines. Crop Sci 38:472-477

Njiti VN, Meksem K, Iqbal MJ, Johnson JE, Kassem MA, Zobrist KF, Kilo VY, Lightfoot DA (2002) Common loci underlie field resistance to soybean sudden death syndrome in Forrest, Pyramid, Essex, and Douglas. Theor Appl Genet 104:294-300

Palmateer AJ, Schmidt ME, Stetina SR, Russin JS (2000) Temperature effects on race determination in Heterodera glycines. J Nematol 32:349-355. 
Prabhu RR, Njiti VN, Johnson JE, Schmidt ME, Klein RJ, Lightfoot DA (1999) Selecting soybean cultivars for dual resistance to cyst nematode sudden death syndrome with two DNA markers. Crop Sci 39:982-987

Qui BX, Arelli PR, Sleper DA (1999) RFLP markers associated with soybean cyst nematode resistance and seed composition in a 'Peking' $\times$ 'Essex' population. Theor Appl Genet 98:356-364

Riggs RD, Schmitt DP (1988) Complete characterization of the race scheme for Heterodera glycines. J Nematol 20: 392-395

Ruben E, Aziz J, Afzal J, Njiti VN, Triwitayakorn K, Iqbal MJ, Yaegashi S, Arelli PR, Town CD, Ishihara H, Meksem K, Lightfoot DA (2006). Genomic analysis of the 'Peking' rhgl locus: Candidate genes that underlie soybean resistance to the cyst nematode. Mol Genet Genome 276: 320-330

Saini N, Shultz JL, Lightfoot DA (2008) Re-annotation of the physical map of Glycine max for ploidy by BAC end sequence driven whole genome shotgun read assembly. BMC Genomics 9: 323-338.

Schmitt DP, Shannon G (1992) Differentiating soybean responses to Heterodera glycines. Crop Sci 32:275-277.

Schuster I, Abdelnoor RV, Marin SRR, Carvalho VP, Kiihl AS, Silva JFV, Sedyama CS, Barros EG, Moreira MA. 2001. Identification of a new major QTL associated with resistance to the soybean cyst nematode (Heterodera glycines). Theor Appl Genet 102:91-96. 
Senda M, Kasai A, Yumoto S, Akada S, Ishikawa R, Harada T, Niizeki M. (2002) Sequence divergence at chalcone synthase gene in pigmented seed coat soybean mutants of the Inhibitor locus. Genes Genet Syst 77:341-350.

Shultz JL, Kurunam D, Shopinski K, Iqbal MJ, Kazi S, Zobrist K, Bashir R, Yaegashi S, Lavu N, Afzal AJ, Yesudas CR, Kassem MA, Wu C, Zhang HB, Town CD, Meksem K, Lightfoot DA (2006a) The Soybean Genome Database (SoyGD): A browser for display of duplicated, polyploid, regions and sequence tagged sites on the integrated physical and genetic maps of Glycine max. Nucleic Acids Res 34:D758-765

Shultz JL, Yesudas CR, Yaegashi S, Afzal J, Kazi S, Lightfoot DA (2006b) Three minimum tile paths from bacterial artificial chromosome libraries of the soybean (Glycine max cv. 'Forrest'): Tools for structural and functional genomics. Plant Methods 2:9-18

Shultz JL, Kazi S, Afzal JA, Bashir R, Lightfoot DA (2007) The development of BACend sequence-based microsatellite markers and placement in the physical and genetic maps of soybean. Theor Appl Genet 114:1081-1090.

Song QJ, Marek LF, Shoemaker RC, Lark KG, Concibido VC, Delannay X, Specht JE, Cregan PB (2004) A new integrated genetic linkage map of the soybean. Theor Appl Genet 109: 122-128

Triwitayakorn K, Njiti VN, Iqbal MJ, Yaegashi S, Town CD, Lightfoot DA (2005) Genomic analysis of a region encompassing $Q R f s 1$ and $Q R f s 2$ : genes that underlie soybean resistance to sudden death syndrome. Genome/Génome 48: 125-138 
Vierling RA, Faghihi J, Ferris VR, Ferris JM (1996) Association of RFLP markers conferring broad-based resistance to the soybean cyst nematode (Heterodera glycines). Theor Appl Genet 92:83-86

Webb, DM, Baltazar BM, Arelli PR, Schupp J, Keim P, Clayton K, Ferreira AR, Owens T, Beavis WD (1995) QTL affecting soybean cyst-nematode resistance. Theor Appl Genet 91:574-581

Webb, DM, Baltazar BM, Arelli PR, Schupp J, Keim P, Clayton K, Ferreira AR, Owens T, Beavis WD (1996) QTL affecting soybean cyst-nematode resistance. United States Patent 5,491,081, Feb 16, 1996

Weisemann JM, Matthews BF, Devine TE (1992) Molecular markers located proximal to the soybean cyst nematode resistance gene Rhg4. Theor Appl Genet 85:136-138

Wrather JA, Anderson TR, Arsyad DM, Gai J, Ploper DL, Portapuglia A, Ram HH, Yorinori JT (1996) Soybean disease loss estimates for the top ten producing countries during. Plant Disease 79:107-110

Wrather JA, Koenning SR, Anderson TR (2003) Effect of diseases on soybean yields in the United States and Ontario (1999 to 2002). Plant Health Progr (online Doi 10.1049)

Wu X, Blake S, Sleper D, Shannon G, Cregan P, Nguyen H (2009) QTL, additive and epistatic effects for SCN resistance in PI 437654. Theor Appl Genet (in press)

Yuan J, Njiti VN, Meksem K, Iqbal MJ, Triwitayakorn K, Kassem MA, Davis GT, Schmidt ME, Lightfoot DA (2002) Quantitative trait loci in two soybean recombinant inbred line populations segregating for yield and disease resistance. Crop Sci 42: 271-277 


\section{Figure legends}

Figure 1. Frequency distributions of the FxH (F5:11) RIL response to SCN HG Type 1.2.5-(race 2) HG Type 1.3-(race 14) and HG Type 0 (race 3). Letters H and F directed by arrows indicate where the mean FI of the Hartwig and Flyer respectively fall within the distribution .

Figure 2. Frequency distribution of FxH19-derived NIL population analyzed for SCN HG Type 1.2.5-female index (FI). Panel A shows NIL 19-1 to 10 ranked by mean FI\%. Female Index below 30\% was moderately resistant; 30-60\% was moderately susceptible; $60-100 \%$ were susceptible and greater than $100 \%$ were highly susceptible. Panel B show the cyst count for each of six plants per NIL 1-10 in numeric order.

Figure 3. Locations of the QTL found in the Flyer by Hartwig population on linkage groups A2, D2 and G for resistance to SCN HG Type 1.2.5-(grey stippled arrows) HG Type 0 (black stippled arrows) and HG Type 1.3 (black solid arrow). The size of the arrow reflects the interval significantly associated by QTL Cartographer or Mapmaker at LOD $>2.5$ or ANOVA at $\mathrm{P}<0.0005$. 
Table 1. Mean Female index plus or minus standard errors of parents, narrow sense heritabilities and probabilities of normality among the FxH $\left(\mathrm{F}_{5: 11}\right)$ RILs for SCN HG Type 1.2.5-, HG Type 0 and HG Type 1.3-(races 2, 3 and 14).

\begin{tabular}{llll}
\hline HG Type & $1.2 .5-$ & 0 & $1.3-$ \\
$($ RILs $)$ & $(\mathrm{n}=14)$ & $(\mathrm{n}=92)$ & $(\mathrm{n}=92)$ \\
\hline Hartwig & $3 \pm 0.4$ & $2 \pm 0.4$ & $2 \pm 0.4$ \\
Flyer & $109 \pm 23$ & $103 \pm 25$ & $123 \pm 33$ \\
FxH (F5:11) & $50.4 \pm 8.35$ & $55.1 \pm 3.97$ & $76.2 \pm 2.36$ \\
Heritability & 0.95 & 0.96 & 0.86 \\
Probability & $<.0001$ & $<.0001$ & $<.0001$ \\
\hline
\end{tabular}


Table 2. Markers and intervals those were associated with resistance to HG Type 0 (race 3) in the FxH RIL population on linkage group G.

\begin{tabular}{lllllllll}
\hline Marker & $P$ value & $\mathrm{R}^{2}(\%)$ & \multicolumn{2}{c}{ FI Means \pm SEM } & \multicolumn{2}{c}{ Intervals } \\
& \multicolumn{9}{c}{ Flyer } & $(\mathrm{n})^{\mathrm{a}}$ & Hartwig & $(\mathrm{n})$ & LOD & QTL var \\
\hline Satt163 & 0.002 & 14 & $64.0 \pm 5.2$ & $(57)$ & $37.6 \pm 5.9$ & $(20)$ \\
Satt275 & 0.005 & 14 & $62.5 \pm 4.6$ & $(47)$ & $34.8 \pm 8.4(33)$ & 3.6 & 18 \\
TMD1 & 0.008 & 11 & $60.8 \pm 4.2$ & $(59)$ & $32.6 \pm 9.1(19)$ & 3.9 & 18 \\
Satt309 & 0.0001 & 22 & $64.3 \pm 4.1$ & $(69)$ & $20.8 \pm 6.8(18)$ & 3.9 & 21 \\
Satt610 & 0.001 & 17 & $66.5 \pm 5.6$ & $(35)$ & $34.2 \pm 6.5(23)$ & 4.9 & 27 \\
\hline
\end{tabular}

${ }^{a}$ Heterogenous lines were excluded

b $\%$ of trait variation explained by each marker-trait association 
Table 3. Markers and intervals that were associated with resistance to HG Type 1.3- (race 14) in the FxH RIL population on linkage group G.

\begin{tabular}{lllllll}
\hline Marker & $P$ value & $\mathrm{R}^{2}(\%)$ & \multicolumn{2}{l}{ FI Means \pm SEM } & \multicolumn{2}{l}{ Intervals } \\
& & & Flyer & Hartwig & LOD & QTL var \\
\hline & & & & & & \\
Satt163 & 0.04 & 6 & $81.2 \pm 2.3$ & $69.1 \pm 5.8$ & \\
Satt309 & 0.001 & 17 & $81.6 \pm 2.1$ & $61.5 \pm 6.9$ & 2.87 & 14.6 \\
\hline
\end{tabular}


Table 4: Four markers that were associated with the QTL underlying resistance to HG Type 1.2.5- segregated in the FxH19-derived near isogenic line (NIL) population. Six plants from each of ten lines within the FxH19-derived NIL population were tested for resistance to HG Type 1.2.5-. There were six plants per genotype. The sixty plants were scored for each of the four markers. Heterozygous lines were excluded

\begin{tabular}{|c|c|c|c|c|c|c|}
\hline Marker & $\underline{\mathbf{L G}}$ & Allele & $\underline{\mathbf{N}}$ & $\underline{\operatorname{Pr}>F}$ & ${ }^{+} \underline{\mathbf{R}^{2}}$ & $\frac{\text { Allelic Mean }}{ \pm \text { SEM }}$ \\
\hline \multirow[t]{2}{*}{ Satt543 } & D2 & $\mathrm{H}$ & 18 & $0.046^{*}$ & 0.19 & $30.8 \pm 7.8$ \\
\hline & & $\mathrm{F}$ & 28 & & & $86.2 \pm 9.4$ \\
\hline \multirow[t]{2}{*}{ Satt594 } & $\mathrm{G}$ & $\mathrm{H}$ & 23 & 0.31 & 0.006 & $58.7 \pm 12.4$ \\
\hline & & $\mathrm{F}$ & 21 & & & $82.4 \pm 8.9$ \\
\hline \multirow[t]{2}{*}{ Satt237 } & $\mathrm{N}$ & $\mathrm{H}$ & 22 & 0.076 & 0.9 & $63.1 \pm 9.8$ \\
\hline & & $\mathrm{F}$ & 31 & & & $84.6 \pm 8.8$ \\
\hline \multirow[t]{2}{*}{ Satt530 } & $\mathrm{N}$ & $\mathrm{H}$ & 25 & 0.12 & 0.7 & $70.6 \pm 9.1$ \\
\hline & & $\mathrm{F}$ & 25 & & & $79.7 \pm 6.2$ \\
\hline
\end{tabular}

${ }^{\ddagger} \mathrm{R}^{2}=$ Sum of squares for given source of variance divided by total sum of squares

$\mathrm{N}=$ Number of individuals scored

* = significance at 0.05 level

$\mathrm{H}=$ Hartwig allele, $\mathrm{F}=$ Flyer allele

Hets and no scores not included 
Table 5: Comparison of the loci underlying resistance to SCN detected in inheritance analysis of PI437654 and Hartwig. Data from were assembled from Patent \# 6,162,967 (Webb et al. 1995); Prabhu et al. (1999); Yuan et al. (2002) and this work. In bold are the loci confirmed by the analysis in FxH. The Rhg gene annotations are from Webb et al. (1995). Rzd is a gene whose allele must match $R h g l$ to prevent zygote death.

\begin{tabular}{|c|c|c|c|c|c|c|}
\hline LG & $\mathrm{A} 2$ & $\mathrm{C} 1$ & G & M & $\mathrm{J}$ & D2 \\
\hline Gene & Rhg4 & Rhg2 & Rhgl & $R z d$ & Rhg5 & Rhg3 \\
\hline \multicolumn{7}{|c|}{ HG Type (race) } \\
\hline $2.5-(1)$ & $\mathrm{x}$ & $\mathrm{x}$ & $\mathrm{x}$ & $\mathrm{x}$ & $\mathrm{x}$ & \\
\hline $1.2 .5-(2)$ & & & $\mathbf{x}$ & $\mathrm{x}$ & $\mathrm{x}$ & $\mathbf{x}$ \\
\hline $0-(3)$ & $\mathbf{x}$ & & $\mathbf{x}$ & $\mathrm{x}$ & & \\
\hline $2-(5)$ & & & $\mathrm{x}$ & $\mathrm{x}$ & $\mathrm{x}$ & \\
\hline $1.3-(14)$ & $\mathbf{x}$ & $\mathrm{x}$ & $\mathbf{x}$ & $\mathrm{x}$ & & \\
\hline
\end{tabular}

